\title{
An Oversampled Channelized UWB Receiver
}

\author{
Lei Feng, Won Namgoong \\ Department of Electrical Engineering \\ University of Southern California \\ leifeng@usc.edu,namgoong@usc.edu
}

\begin{abstract}
To digitize the ultra-wideband (UWB) signal at its Nyquist rate, a frequency channelized receiver for UWB radio based on hybrid filter banks (HFB) is presented. Among the challenges of such receivers are the uncertainties of the analog analysis filters and the slow convergence speed. To overcome these problems, a channelized receiver operating at slightly above the critically sampling rate is presented. The proposed receiver, which is designed for use in transmitted reference (TR) systems, combines the synthesis filters and the matched-filter so that the combined response of the analysis filter and the propagation channel is estimated. The resulting convergence speed is comparable to an ideal full band receiver. In addition, all of the receiver functions are performed at the reduced subband sampling rate, relaxing the requirements on the digital circuitries.
\end{abstract}

\section{INTRODUCTION}

Ultra-wideband (UWB) systems can coexist with narrow-band radio systems by spreading the signal energy over a very wide bandwidth [1]. A major chanllenge in UWB communication is achieving accurate channel estimation. Transmitted reference (TR) modulation schemes has been proposed because of the ease of channel estimation in a dense multipath enviroment [2]. Optimal detection for UWB TR system was investigated in [3]. To achieve high performance, the UWB receiver needs to digitize the UWB signal at least at the signal Nyquist rate (usually several gigahertz) so that sophisticated digital detection schemes can be applied. Since designing a single analog-to-digital (ADC) to operate at such sampling frequencies is difficult, parallel ADC architectures with each ADC operating at a fraction of the effective sampling frequency need to be employed.

To sample at a fraction of the effective sampling frequency, a frequency channelized receiver based on hybrid filter banks (HFB) (i.e., continuous-time analysis filters and discrete-time synthesis filters) has been proposed in [4] and [5]. Among the advantages of the frequency channelized

This work was supported in part by the Army Research Office under contract number DAAD19-01-1-0477 and National Science Foundation under contract number ECS0134629 . receiver compared to the more conventional time channelized (i.e., time-interleaved ADC) receivers are the ease of designing the sample/hold circuitries, greater robustness to jitter/phase noise, and reduced ADC dynamic range requirements [4].

In the frequency channelized receiver, signal detection can be achieved by first reconstructing the channelized signal then processing the sampled full band signal as in the conventional full channel receiver. Design of perfect reconstruction (PR) or approximately PR HFBs have been explored [6][7]. A potential problem with this approach is that designing the digital synthesis filters requires accurate knowledge of the transfer functions of the analog analysis filters, which may be unavailable in practice because of the variations resulting from temperature and fabrication process uncertainties.

The distortion caused by the propagation channel and the analysis filters can be compensated by employing adaptive synthesis filters to recover the transmitted signals. The primary drawback is the slow convergence speed as shown in [8] and [9]. Oversampled filter banks have also been proposed to achieve faster convergence [10]. However, the convergence speed is still not sufficiently fast in time-varying UWB channels.

The proposed oversampled UWB receiver differs from existing HFBs in that the objective is not to reconstruct the received signal but to detect the transmitted symbols. Instead of determining the synthesis filters that reconstruct the received full band signal waveform, which suffers from slow convergence, the proposed receiver performs signal detection by estimating the combined responses of the analysis filter and the propagation channel. If the analysis filters are designed to satisfy the power complementary condition, which can be achieved as described in a later section, the PR synthesis filters are the time-reversed complex conjugates of the analysis filters [11]. Since the matched filter is also the time-reversed complex conjugate of the propagation channel, the matched filter and the synthesis filter can be combined and the estimate of the optimal pulse response for detection can be obtained by simply averaging the combined responses of the propagation channel and the analysis filters.

This paper is organized as follows. Section 2 introduces the system model. Section 3 presents the proposed receiver 
structure based on the PR filter bank. Simulation results are provided in Section 4 and conclusions are drawn in Section 5.

\section{SYSTEM MODEL}

The UWB signal is a time-hopping pulse train. To simplify the problem, we assume that the pulse position in each frame is fixed, a symbol is transmitted in each frame, and sufficient guard period is introduced to prevent interference between frames. The received UWB signal $r(t)$ is

$$
r(t)=\sum_{k} a_{k} p(t-k T)+v(t)
$$

where $a_{k}$ is the $k$ th transmitted antipodal symbol, $T$ is the frame period, $p(t)$ is the received signal pulse, and $v(t)$ is the additive white gaussian noise (AWGN). In this paper, $k$ is a finite number since a block of frames is transmitted in TR systems.

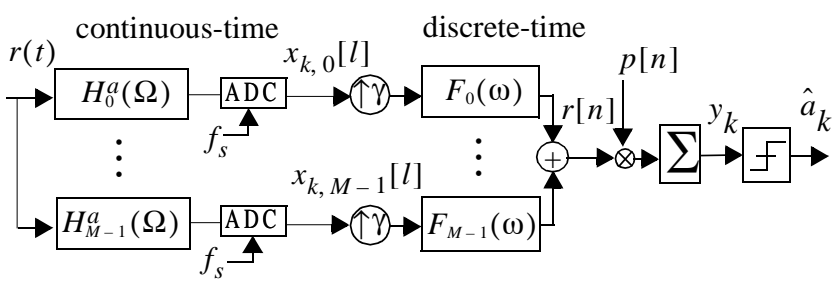

Figure $1:$ A receiver with a PR filter bank

A HFB receiver is shown in Figure 1. The received signal $r(t)$ is passed through a bank of continuous-time analysis filters $\left\{H_{m}^{a}(\Omega)\right\}$ with $m \in\{0,1, \ldots, M-1\}$, sampled and digitized by the ADC at a rate of $1 / T_{s}$, upsampled by $\gamma$, filtered by the synthesis filters $\left\{F_{m}(\omega)\right\}$, then summed to reconstruct the received signal. The effective sampling frequency of the HFB is $1 / T_{e}$ and is related to the ADC sampling frequency $1 / T_{s}$ by $\gamma$, i.e., $T_{e}=T_{s} / \gamma$. Assuming that $\left\{H_{m}^{a}(\Omega)\right\}$ and $\left\{F_{m}(\omega)\right\}$ achieve perfect reconstruction (PR), the reconstructed signal $r[n]$ is correlated with the sampled pulse response $p[n]\left(=p\left(n T_{e}\right)\right)$ then passed through a slicer to achieve optimal detection. frame is

The $l$ th sample of the $m$ th subband channel in the $k$ th

$$
x_{k, m}[l]=\int_{-\infty}^{\infty} h_{m}^{a}\left(k T+l T_{s}-\tau\right) r(\tau) d \tau
$$

where $h_{m}^{a}(t)$ is the impulse response of the $m$ th analysis filter $H_{m}^{a}(\Omega)$. To obtain the discrete equivalent model of the HFB receiver in Figure 1, we assume that $\left\{H_{m}^{a}(\Omega)\right\}$ and $r(t)$ are approximately bandlimited within $1 /\left(2 T_{e}\right)$. The AWGN $v(t)$ can be assumed with little loss in accuracy to have a brickwall power spectral density (PSD) of bandwidth $1 /\left(2 T_{e}\right)$, since $\left\{H_{m}^{a}(\Omega)\right\}$ removes most of the frequency components above $1 /\left(2 T_{e}\right)$. Applying the equivalence theo- rem of digital and analog signal processing [12], (2) becomes

$$
\begin{aligned}
x_{k, m}[l] & =T_{e} \sum_{\substack{i=-\infty \\
\infty}}^{\infty} h_{m}^{a}\left(l T_{s}-i T_{e}\right) r\left(k T_{s}+i T_{e}\right) \\
& =\sum_{i=-\infty} h_{m}[l \gamma-i] r\left[k N_{e}+i\right]
\end{aligned}
$$

where $h_{m}[n]=T_{e} h_{m}^{a}\left(n T_{e}\right)$ and

$$
r[n]=\sum_{k} a_{k} p\left(n T_{e}-k T\right)+v[n]
$$

In (4), $v[n]$ is the AWGN with a PSD that corresponds to that of $v(t)$.

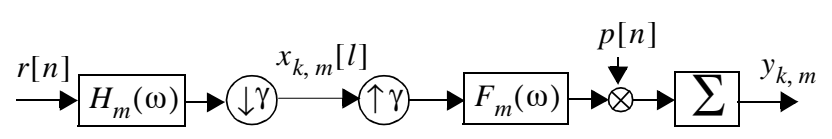

Figure 2 : Correlation in the $m$ th subband.

Figure 2 shows the equivalent discrete model of the $m$ th subband channel in Figure 1 with the correlation operation pushed to before the subband summation operation. In the equivalent model, the continuous-time analysis filter $H_{m}^{a}(\Omega)$ is replaced by a discrete-time filter $H_{m}(\omega)$, whose impulse response is $h_{m}[n]$.

Substituting $r[n]$ in (3) with (4), the ADC output in the $m$ th subband channel can be written as

$$
x_{k, m}[l]=a_{k} p_{m}[l]+v_{k, m}[l]
$$

where

$$
\begin{gathered}
p_{m}[l]=\sum_{n} h_{m}[\gamma l-n] p[n] \\
v_{k, m}[l]=\sum_{n} h_{m}[\gamma l-n] v[n]
\end{gathered}
$$

After some straightforward mathematical manipulations, the subband correlation output $y_{k, m}$ can be shown to be

$$
y_{k, m}=\sum_{l} x_{k, m}[l]\left(\sum_{n} f_{m}[n-\gamma l] p[n]\right)
$$

where $f_{m}[n]$ is the impulse response of $F_{m}(\omega)$. The input to the slicer is the summation of all the outputs of the equivalent subband channels, i.e.,

$$
y_{k}=\sum_{m=0}^{M-1} y_{k, m}
$$

(8) and (9) suggest that detection in a HFB receiver can be achieved at the ADC sampling rate by appropriately correlating the ADC output then summing. Since no upsampling operation is needed after the ADC, all of the receiver functions are performed at the ADC sampling rate. This is unlike 
a straightforward implementation of the PR filter bank receiver that operates at the effective sampling frequency. The reduced operating frequency in the proposed receiver, therefore, relaxes the requirements on the digital circuitries.

\section{THE PROPOSED RECEIVER}

\subsection{Optimal detection and signal estimation}

Optimal detection in the HFB receiver can be achieved by employing a PR filter bank. The PR conditions are:

$$
\begin{gathered}
\sum_{m=0}^{M-1} H_{m}(\omega) F_{m}(\omega)=\gamma \\
\sum_{m=0}^{M} H_{m}(\omega-2 \pi v / \gamma) F_{m}(\omega)=0
\end{gathered}
$$

for $v=1, \ldots, \gamma-1$ and all $\omega$. The difficulty of achieving PR in a HFB results from the uncertainties in the transfer function of the analog analysis filter $H_{m}(\omega)$. As described in the following subsection, however, accurate control of the transfer function amplitude is possible by introducing an additional digital filter and operating the HFB slightly above the critically sampling rate.

To simplify the estimation process, the term in the parentheses of (8) and the subband signal pulse response in (6) can be made to be complex conjugates of each other by designing $F_{m}(\omega)$ to be

$$
f_{m}[n]=h_{m} *[-n]
$$

The PR conditions then become

$$
\begin{gathered}
\sum_{m=0}^{M-1}\left|H_{m}(\omega)\right|^{2}=\gamma \\
\sum_{m=0}^{M-1} H_{m}(\omega-2 \pi v / \gamma) H_{m} *(\omega)=0
\end{gathered}
$$

The resulting subband correlation output $y_{k, m}$ is

$$
y_{k, m}=\sum_{l}\left(a_{k} p_{m}[l]+v_{k, m}[l]\right) p_{m} *[l]
$$

From (15), the advantage of designing $F_{m}(\omega)$ as in (12) becomes clear. The task of determining the optimum correlator is to simply estimate the subband pulse response $p_{m}[l]$ based on the corresponding ADC samples $x_{k, m}[l]$. This estimation can be readily obtained by simply averaging out $x_{k, m}[l]$ with the data modulation removed by operating in either data aided or decision directed modes.

\subsection{Subband filters}

Instead of using a bank of bandpass filters for $H_{m}(\omega)$, the frequency channelization can be achieved by employing a bank of complex mixers and lowpass filters [11]. The advantages of this channelization scheme are two-fold: the design of sharp bandpass filters centered at high frequencies, which are difficult to realize in an integrated receiver, is avoided, and the ADC sample/hold circuitries are relaxed, since the ADC only sees the bandwidth of the lowpass filter.

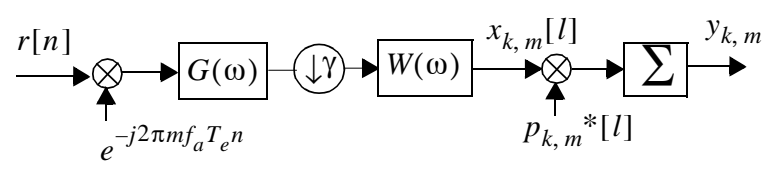

Figure 3 : The modulation model and subband filter

The $m$ th subband channel employing such channelization approach is shown in Figure 3. The mixer downconversion frequency of the $m$ th subband channel is $m f_{a}$, and the lowpass filter, which is the same for all subband channels, is $G(\omega)$. To remove the dependency on the analog filter $G(\omega)$, a digital filter $W(\omega)$ is introduced. The effect of $W(\omega)$ on the transfer function of the analysis filter is best understood by pushing $W(\omega)$ to before the decimator and pushing the mixer to after the filters as shown in Figure 4. In this equivalent model, $G_{m}(\omega)=G\left(\omega-m \omega_{a} T_{e}\right) \quad$ and $W_{m}(\omega)=W\left(\gamma \omega-m \omega_{a} T_{e}\right)$, where $\omega_{a}=2 \pi f_{a}$. The analysis filter can now be viewed as being

$$
H_{m}(\omega)=G_{m}(\omega) W_{m}(\omega)
$$

The mixer after the decimator can be absorbed by the correlator.

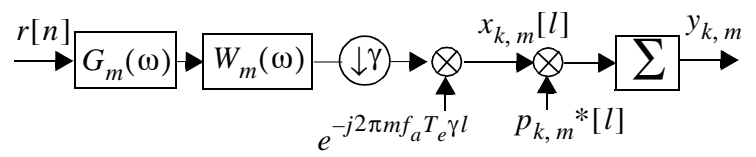

Figure 4 : Changing the operation order

$W(\gamma \omega)$ consists of multiple images due to upsampling.

$$
W(\gamma \omega)=\sum_{v=0}^{\gamma-1} W^{p}\left(\omega-\frac{2 \pi v}{\gamma}\right)
$$

where $W^{p}(\omega)$ is a lowpass filter. Since we are interested in $W^{p}(\omega)$ only, $G(\omega)$ is designed to be sharp enough to eliminate the other images as shown in Figure 5. The analysis filter can now be viewed as being

$$
H_{m}(\omega)=G_{m}(\omega) W^{p}\left(\omega-m \omega_{a} T_{e}\right)
$$

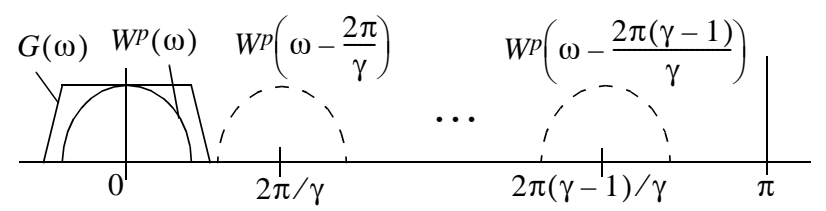

Figure 5 : Filter images in the equivalent model

To satisfy the second PR condition given in (14), the filter bank should be sufficiently oversampled and $H_{m}(\omega)$ 
made adequately sharp to remove all of its spectral images. This is equivalent to designing $W^{p}(\omega)$ so that the overlap between its images is negligible. Once $W^{p}(\omega)$ is designed, $W(\omega)$ can be obtained by decimation.

In this channelization approach, the first PR condition given in (10) and (13) is

$$
\sum_{m=0}\left|G_{m}(\omega)\right|^{2}\left|W^{p}\left(\omega-m \omega_{a} T_{e}\right)\right|^{2}=\gamma
$$

To remove the uncertainty in $G_{m}(\omega)$, we make the magnitude of $G_{m}(\omega)$ flat within the passband of $W^{p}\left(\omega-m \omega_{a} T_{e}\right)$. The PR condition then becomes a function of only the digital filter $W^{p}(\omega)$, which can be accurately controlled, i.e.,

$$
\sum_{m=0}^{M-1}\left|W^{p}\left(\omega-m \omega_{a} T_{e}\right)\right|^{2}=\gamma
$$

\subsection{The Proposed Channelized Receiver}

The proposed channelized receiver is shown in Figure 8. As described in the previous subsection, the analysis filters are realized by a bank of equally spaced mixers and identical lowpass filters $G(\Omega)$. A digital filter $W(\omega)$ is also included to control the transfer function of the analysis filters. Since the received signal $r(t)$ is real, the filter banks operate only on the positive frequency spectrum except for the zeroth subband channel, which operates on both the positive and the negative frequencies. Signal detection, therefore, is performed after scaling the output of the nonzero subband channels by two and taking the real part of the summation.

The proposed oversampled HFB receiver detects the received signal by correlating the ADC output $x_{k, m}[l]$ by $p_{m}{ }^{*}[l]$ as described in (15). In practice, however, $p_{m}{ }^{*}[l]$ is unknown at the receiver and must be estimated based on $x_{k, m}[l]$. In TR system, the estimation can be achieved by simply averaging out $x_{k, m}[l]$ corresponding to the reference symbols after removing the effect of the downconversion mixer.

\section{SIMULATION RESULTS}

The transmitted monocycle is the second derivative of a gaussian pulse, i.e.,

$$
p(t)=\left(1-4 \pi t^{2}\right) \exp \left(-2 \pi t^{2}\right)
$$

The received noise free signal pulse is a superposition of monocycles with different delays and amplitudes. The multipath model used is the CM1 channel model recommended by the IEEE P802.15-02/368r5-SG3a. The sampling period is $T_{s}=0.167 n s$.

For accurate simulation, the continuous-time signals and the analog filters are modeled by discretizing at twice the effective sampling frequency. The downsampling rate in the previous sections is $2 \gamma$ instead of $\gamma$. Assuming $M=4$ and $\gamma=5$, the effective sampling rate is $7 / 5$ times the critically sampling rate. The low pass filter $G(\Omega)$ in the analysis filters is an eighth order Butterworth filter, and its $3 \mathrm{~dB}$ frequency is at $1 /\left(12 T_{e}\right) . W(\omega)$ is a ten tap filter and is obtained from [13] [14].

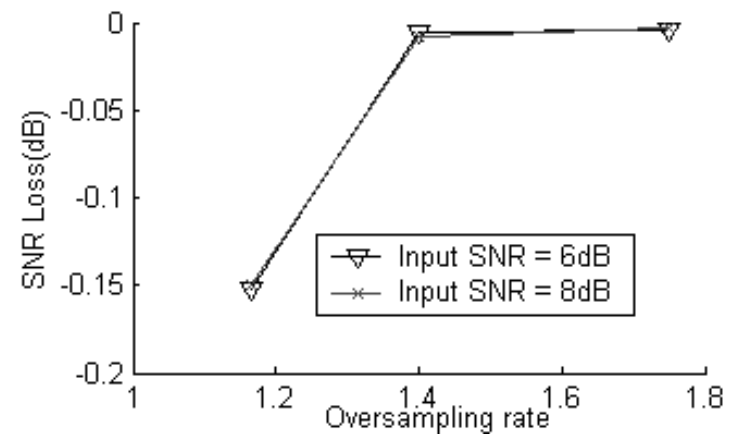

Figure 6 : SNR loss v.s. Oversampling rate

Figure 6 shows the SNR loss of the channelized receiver at the input of the slicer at different oversampling rate, which is defined as the effective sampling rate ratio between the oversampled receiver and the critically sampled receiver. $p_{m}{ }^{*}[l]$ is assumed to be known. The SNR loss is present because the practical analysis filters do not completely satisfy the PR requirements (i.e. remaining images of the discrete filters, aliasing due to the analog filters, and nonflat amplitude of the analog filters whithin the required band). The SNR loss is negligible with moderate oversampling rate.

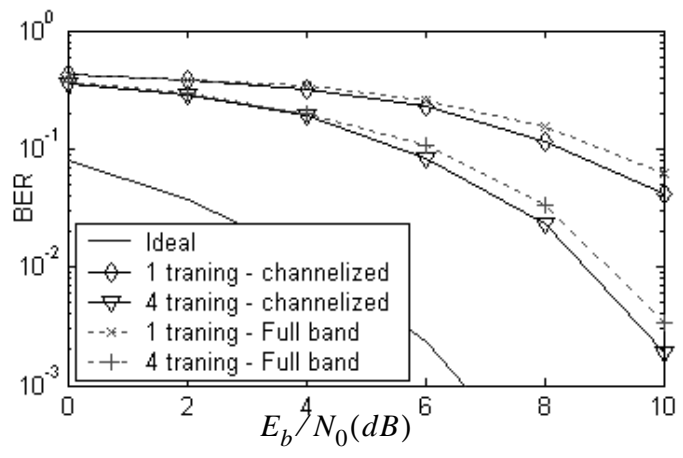

Figure 7 : BER comparison

Figure 7 shows the bit error rate (BER) for a TR system when $p_{m}{ }^{*}[l]$ is unknown. The first 1 or 4 training symbols in a block of 100 symbols is used to estimate the time varying $p_{m}{ }^{*}[l]$. The estimation is then used to detect the remaining symbols in the block. The performance of an ideal matched filter receiver with perfect knowledge of the received signal pulse is shown as the lower bound. A full band receiver, which samples the UWB signal with a single ADC at the effective sampling rate and estimates the signal pulse by 
averaging the training symbols, is used for comparison. As the number of training symbols increases, the performance of both receivers approach that of the ideal receiver.

\section{CONCLUSION}

An oversampled channelized receiver for UWB radio TR systems is proposed. By combining the PR synthesis filters and the matched filter, the optimal detection is achieved by estimating the combined responses of the channelizer and the propagation channel. The proposed receiver achieves performance comparable to that of an ideal full band receiver.

\section{REFERENCES}

[1] M. Z. Win and R. A. Scholtz, "Impulse Radio: How It Works," IEEE Comm. Letters, vol. 2, NO. 2, pp. 36-38, Feb. 1998

[2] R. Hoctor and H. Tomlison, "An Overview of DelayHopped, Transmitted-Reference RF Communications," GE Research and Development Center, Tech. Rep. 2001CRD198, 2002

[3] S. Franz and U. Mitra, "On Optimal Data Detection for UWB Transmitted Reference Systems," IEEE GLOBECOM 2003, pp744-748, 2003

[4] W. Namgoong, "A Channelized Digital Ultra-Wideband Receiver," IEEE Trans. Wireless Comm., vol. 2, pp. 502510, May 2003

[5] W. Namgong, "Channelized Digital Receivers for Impulse Radio,” ICC '03. , Volume: 4 , 2003 , pp. 2884 2888

[6] S. R. Velazquez, T. Q. Nguyen and S. R. Broadstone, "Design of Hybrid Filter Banks for Analog/Digital Conversion," IEEE Trans. on Signal Processing, vol. 46 NO. 4, Apr. 1998
[7] P. Lowenborg, H. Johansson and L. Wanhammar, "TwoChannel Digital and Hybrid Analog/Digital Multirate Filter Banks With Very Low-Complexity Analysis or Synthesis Filters," IEEE Trans. on Circuits and Systems II: Analog and Digital Signal Processing, vol. 50, NO. 7, Jul. 2003

[8] A. Gilloire and M. Vetterli, "Adaptive Filtering in Subbands with Critical Sampling: Analysis, Experiments, and Application to Acoustic Echo Cancellation," IEEE Trans. on Signal Proc. vol. 40, NO. 8, Aug. 1992

[9] L. Feng and W. Namgoong, "A frequency channelized adaptive wideband receiver for high-speed links ," Signal Processing Systems, 2003. SIPS 2003. IEEE Workshop on , 27 - 29 Aug. 2003, pp. 24 -28

[10] P. L. De Leon II and D. M. Etter, "Experimental Results with Increased Bandwidth Analysis Filters in Oversampled, Subband Acoustic Echo Cancelers," IEEE Signal Processing Letters, vol. 2, NO.1, Jan. 1995

[11] R. E. Crochire and L. R. Rabiner, "Multirate Digital Signal Processing," Prentice Hall, 1983

[12] H. Meyr, M. Moeneclaey and S. A. Fehtel, "Digital Communication Receivers: Synchronization, Channel Estimation, and Signal Processing," John Wiley \& Sons, Inc. 1998

[13] S. Weiss, A. Stenger, R. W. Stewart, and R. Rabenstein, "Steady-State Performance Limitations of Subband Adaptive Filters," IEEE Trans. on Signal Processing, Vol.49, No.9, pp.1982-1991, Sep. 2001

[14] M. Harteneck, S. Weiss, and R.W. Stewart, "Design of Near Perfect Reconstruction Oversampled Filter Banks for Subband Adaptive Filters," IEEE Trans. on Circuits and Systems Part II: Analog and Digital Signal Processing, Vol.46, No.8, pp.1081-1086, Aug. 1999

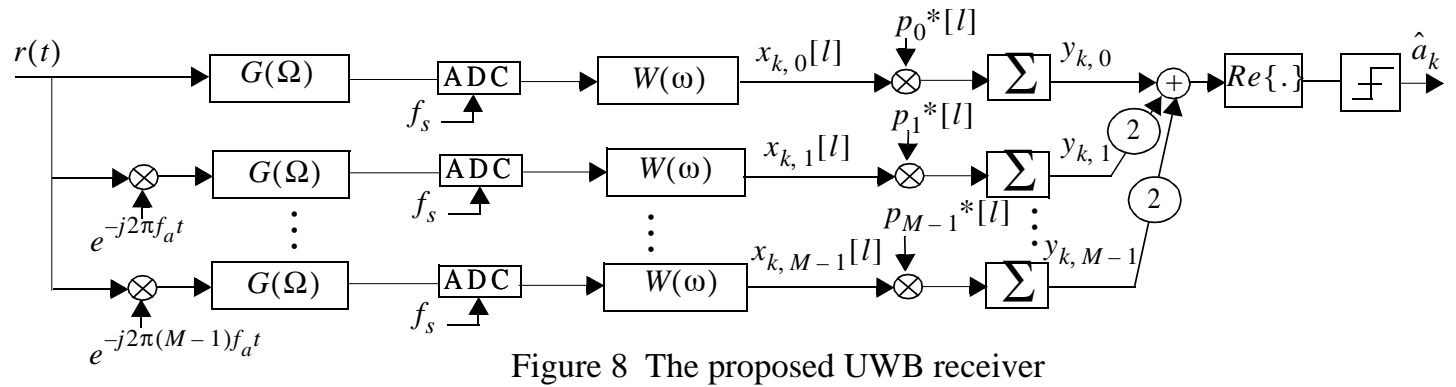

